\title{
Phosphoglycerate kinase 1 promotes radioresistance in U251 human glioma cells
}

\author{
HAO DING $^{1}$, YI-JUN CHENG ${ }^{1}$, HUA YAN ${ }^{1}$, RUI ZHANG ${ }^{1}$, JIN-BING ZHAO $^{1}$, \\ CHUN-FA QIAN $^{1}$, WEN-BIN ZHANG ${ }^{1}$, HONG XIAO $^{2}$ and HONG-YI LIU ${ }^{1}$ \\ ${ }^{1}$ Department of Neurosurgery and ${ }^{2}$ Neuro-Psychiatric Institute, Nanjing Medical University, \\ Affiliated Nanjing Brain Hospital, Nanjing, Jiangsu, P.R. China
}

Received October 23, 2013; Accepted November 18, 2013

DOI: 10.3892/or.2013.2874

\begin{abstract}
Phosphoglycerate kinase 1 (PGK1) has been found to be increased in radioresistant astrocytomas. The present study was designed to investigate the potential role of PGK1 in the radioresistance in U251 human cells. Quantitative PCR and western blot analysis were performed to evaluate PGK1 expression for mRNA levels and protein levels, respectively. The short hairpin RNA (shRNA)-PGK1 and the high expression plasmids were transfected to radioresistant U251 cells (RR-U251 cells) or normal U251 cells using Lipofectamine ${ }^{\mathrm{TM}}$ 2000. The cell viability was determined by MTT assay. The wound-healing assay (WHA) was used to evaluate cell migration ability. Cell invasion abilities were examined using a Transwell culture chamber system. Our results showed that the expression of PGK1 was significantly increased in RR-U251 cells compared to normal U251 cells. Following irradiation, the cell viability as well as the migration and invasion ability were significantly higher in RR-U251 cells compared with normal U251 cells. Downregulating PGK1 using shRNA induced a significantly downregulated cell viability and decreased migration and invasion ability, and overexpression of PGK1 contributed to upregulated cell viability and increased migration and invasion ability, both in RR-U251 cells and normal U251 cells. These findings suggest that PGK1 could promote radioresistance in $\mathrm{U} 251$ human cells.
\end{abstract}

\section{Introduction}

Glioma, one of the most aggressive malignancies, is the most common primary brain tumor in adults, accounting for $80 \%$

Correspondence to: Dr Hong-Yi Liu, Department of Neurosurgery, Nanjing Medical University, Affiliated Nanjing Brain Hospital, 264 Guangzhou Road, Nanjing, Jiangsu 210029, P.R. China

E-mail: hyliu18@126.com

Dr Hong Xiao, Neuro-Psychiatric Institute, Nanjing Medical University, Affiliated Nanjing Brain Hospital, 264 Guangzhou Road, Nanjing, Jiangsu 210029, P.R. China

E-mail: xhnkyy123@163.com

Key words: phosphoglycerate kinase 1, radioresistance, glioma, U251 cells of primary malignant brain tumors $(1,2)$. Astrocytoma presents the most common type. To date, despite major research efforts and progress in therapy, including neurosurgical technology, radiation therapy, chemotherapy and molecular therapy, the prognosis of gliomas remains poor. Patients with malignant glioma have a median survival rate of only 15 months (3-5). Excessive proliferation, resistance to apoptotic stimuli, neovascularization, disseminated tumor growth and suppression of antitumor immune surveillance are key biological features that contribute to the malignant phenotype of gliomas $(6,7)$.

Maximal surgical resection of the tumor mass, combined with chemotherapy and/or radiotherapy, is the standard of care $(8,9)$. However, chemotherapy has been hampered due to resistance of these tumor cells to available agents and challenges in delivering agents targeting the tumor cells. Gliomas often respond to radiotherapy, but subsequent recurrence is almost inevitable, which suggests insufficient ability to kill the tumorigenic cells.

Radioresistance is a widespread challenge in malignant glioma therapy (10). In a previous study from our laboratory, we found that phosphoglycerate kinase 1 (PGK1) was significantly upregulated in radioresistant astrocytomas (11), which suggested that PGK1 may be involved in the radioresistant phenotype and may be a potential biomarker for developing better therapeutic methods.

PGK1 is an ATP-generating glycolytic enzyme in the glycolytic pathway catalyzing the conversion of 1,3-diphosphoglycerate to 3-phosphoglycerate and further affects the DNA replication and repair (12). It was previously shown that the increased expression of PGK1 was detected in colon cancer tissue from metastatic patients (13), lung adenocarcinoma (14), gastric (15), prostate (16), pancreatic (17), and multidrug resistant ovarian cancer, but was moderate in adjacent non-tumor tissues (18) and resistant in ovarian cancer cells (19).

In view of the involvement of PGK1 in malignant tumors, in the present study, U251 cells, a human glioma cell line, were selected as our in vitro model to investigate whether PGK1 is involved in the radioresistance. Our results showed that an increased PGK1 expression was observed in radioresistant $\mathrm{U} 251$ cells, and it could promote radioresistance in U251 human glioma cells. 


\section{Materials and methods}

Cell culture. Human glioma U251 cells, obtained from Nanjing KeyGen Biotech. (Co. Ltd., Nanjing, China) were cultured in Dulbecco's modified Eagle's medium (DMEM; Gibco-Invitrogen, Carlsbad, CA, USA) supplemented with $10 \%$ fetal calf serum (Gibco-Invitrogen), $100 \mathrm{U} / \mathrm{ml}$ penicillin and $100 \mu \mathrm{g} / \mathrm{ml}$ streptomycin. Cells were incubated at $37^{\circ} \mathrm{C}$ in a humidified incubator containing $5 \% \mathrm{CO}_{2}$.

Establishment of radioresistant U251 cells (RR-U251). U251 cells were irradiated as monolayer culture by the $\gamma$-rays of a ${ }^{60} \mathrm{Co}$ source at $0.5 \mathrm{~Gy} / \mathrm{min}$ for $10 \mathrm{~min}$ per time until the accumulation was $60 \mathrm{~Gy}(20)$.

Transfection. The shRNA-PGK1 plasmid and the high expression plasmid pcDNA3.1-PGK1 were synthesized by Shanghai GenePharma Co., Ltd. (Shanghai, China). shRNA-PGK1 was designed to target the coding region of the human PGK1 sequence (5'-GCAAGGATGTTCTGTTCTTGA-3'; Gene ID:5230). A negative control (5'-GTTCTCCGAACGTGTC ACGT-3') was also obtained from Shanghai GenePharma.

When U251 cells in 6-well plates were grown to 70-80\% confluence, $4 \mu \mathrm{g}$ shRNAs-PGK1 or the high expression plasmids and $8 \mu 1$ Lipofectamine ${ }^{\mathrm{TM}} 2000$ (Invitrogen) were diluted in $240 \mu$ l Opti-MEM medium separately, after 5-min standing, the diluted plasmids and Lipofectamine 2000 were mixed and added into each well after 20 min standing (final concentration for plasmids was $2 \mu \mathrm{g} / \mathrm{ml}$ ). Six hours after transfection, the medium was replaced with fresh DMEM containing 10\% fetal bovine serum (FBS), and the cells were incubated for an additional $18 \mathrm{~h}$ ( $24 \mathrm{~h}$ after transfection) for radiation treatment.

Total RNA extraction and real-time polymerase chain reaction (real-time PCR). Total RNA was extracted in each group (normal U251 cells and established RR-U251 cells) with SV Total RNA Isolation system (Promega, Madison, WI, USA) according to the manufacturer's protocol. The total RNA was then reverse transcribed to cDNA with reverse transcription System (Promega). mRNA expression was determined by real-time PCR using GoTaq ${ }^{\circledR}$ qPCR Master Mix under standard thermocycler conditions (Eppendorf AG22331, Hamburg, Germany).

Sequences of PCR primer pairs were: human GAPDH, forward, 5'-CGCTGAGTACGTCGTGGAGTC-3' and reverse, 5'-GCTGATGATCTTGAGGCTGTTGTC-3'. Human PGK1, forward, 5'-ATGCTGAGGCTGTCACTCGG-3' and reverse, 5'-CACAGCAAGTGGCAGTGTCTCC-3'.

The PCR amplification began with an initial denaturation step at $95^{\circ} \mathrm{C}$ for $2 \mathrm{~min}$ followed by 40 cycles at $95^{\circ} \mathrm{C}$ for $45 \mathrm{sec}$, $58^{\circ} \mathrm{C}$ for $45 \mathrm{sec}$, and $72^{\circ} \mathrm{C}$ for $45 \mathrm{sec}$. Relative gene expression values were determined using the $2^{-\Delta \Delta \mathrm{CT}}$ method as previously described (21). The human GAPDH gene was used as an endogenous control for sample normalization. Results were presented as fold increases relative to the expression of human GAPDH.

Western blot analysis. U251 cells were washed with cold phosphate-buffered saline (PBS) three times and then scraped from the well. The cells were lysed with lysis buffer (Thermo Fisher
Scientific, Runcorn, UK). The cell lysate was centrifuged and the supernatant was collected. All samples were diluted in loading buffer (Nanjing Sunshine Biotechnology, Co., Ltd., Nanjing, China) and boiled for $3 \mathrm{~min}$. Samples (30 $\mu \mathrm{g})$ were loaded on a $10 \%$ sodium dodecyl sulfate-polyacrylamide gel by electrophoresis (SDS-PAGE). Prestained markers were used to estimate molecular weight. Proteins were transferred to polyvinylidene difluoride membranes (Nanjing Sunshine Biotechnology), blocked by $5 \%$ skim milk and incubated overnight at $4^{\circ} \mathrm{C}$ with antibodies from mouse against human PGK1 diluted in TBST buffer (1:1,000; Abcam, Cambridge, MA, USA), followed by a goat anti-mouse horseradish peroxidaseconjugated secondary antibody at $37^{\circ} \mathrm{C}$ for $1 \mathrm{~h}(1: 10,000)$. The membranes were washed three additional times with TBST, and antibody-antigen complexes were revealed using an enhanced chemiluminescent (ECL) technique, using a SuperSignal West Pico Trial kit obtained from Thermo Fisher Scientific according to the manufacturer's instructions. The membrane was stripped and reprobed with a primary antibody against $\alpha$-tubulin (1:400 dilution; Nanjing Sunshine Biotechnology) as a control. Quantitation was performed by computer with commercial software (ImageQuan Molecular Dynamics, Sunnyvale, CA, USA).

Cell viability assay. The cell viability was determined by the MTT [3-(4,5-dimethylthiazol)-2,5-diphenyltetrazolium bromide] assay (Sigma-Aldrich, St. Louis, MO, USA). Briefly, with or without radiation by ${ }^{60} \mathrm{Co}$, the normal U251 cells and RR-U251 cells $5 \times 10^{3}$ cells/well were seeded in 96-well plates and allowed to attach for $48 \mathrm{~h}$. Each group contained eight wells. MTT $(20 \mu \mathrm{l})$ was added to each well and the cells were incubated at $37^{\circ} \mathrm{C}$ for $4 \mathrm{~h}$. The reaction was then stopped by adding $150 \mu \mathrm{l}$ of dimethyl sulfoxide (DMSO) for $10 \mathrm{~min}$. Optical density (OD) was obtained at a wavelength of $570 \mathrm{~nm}$ using a microplate reader. Viability was calculated by the following equation: Cell viability $(\%)=100 \times$ (OD treatment/OD control) $\%$.

Cell migration assay. The wound-healing assay (WHA) method was used to evaluate cell migration ability. The normal U251 and RR-U251 cells, treated with shRNA-PGK1, overexpression PGK1 or untreated, were cultured in 6-well plates until $\sim 80-90 \%$ confluence was reached. The monolayer cells, with or without radiation by ${ }^{60} \mathrm{Co}$, were scratched out using the tip of a $10 \mu \mathrm{l}$ pipette to create the wound line. To monitor the migration, images were captured with a microscope at 0 and $24 \mathrm{~h}$ at the same site, and the migration distance was measured. The ratio of the increased area by cell migration after $24 \mathrm{~h}$ to that at $0 \mathrm{~h}$ was calculated to quantitate the extent of migration. Migration ratio $=\left(\right.$ Width $\left._{0 \mathrm{~h}}-\mathrm{Width}_{24 \mathrm{~h}}\right) / \mathrm{Width}_{0 \mathrm{~h}} \times 100 \%$.

Cell invasion assessment. Cell invasion abilities were examined using a 24-well Transwell culture chamber system. The filter membrane with $8-\mu \mathrm{m}$ pores was coated with Matrigel (1:8 diluted in DMEM medium; BD Biosciences, Franklin Lakes, NJ, USA). The normal U251 and RR-U251 cells, treated with shRNA-PGK1, overexpression PGK1 or untreated, were cultured in 6-well plates until confluence was reached. The monolayer cells, before irradiation or $6 \mathrm{~h}$ after radiation by ${ }^{60} \mathrm{Co}$, were trypsinized and resuspended in serum-free DMEM 

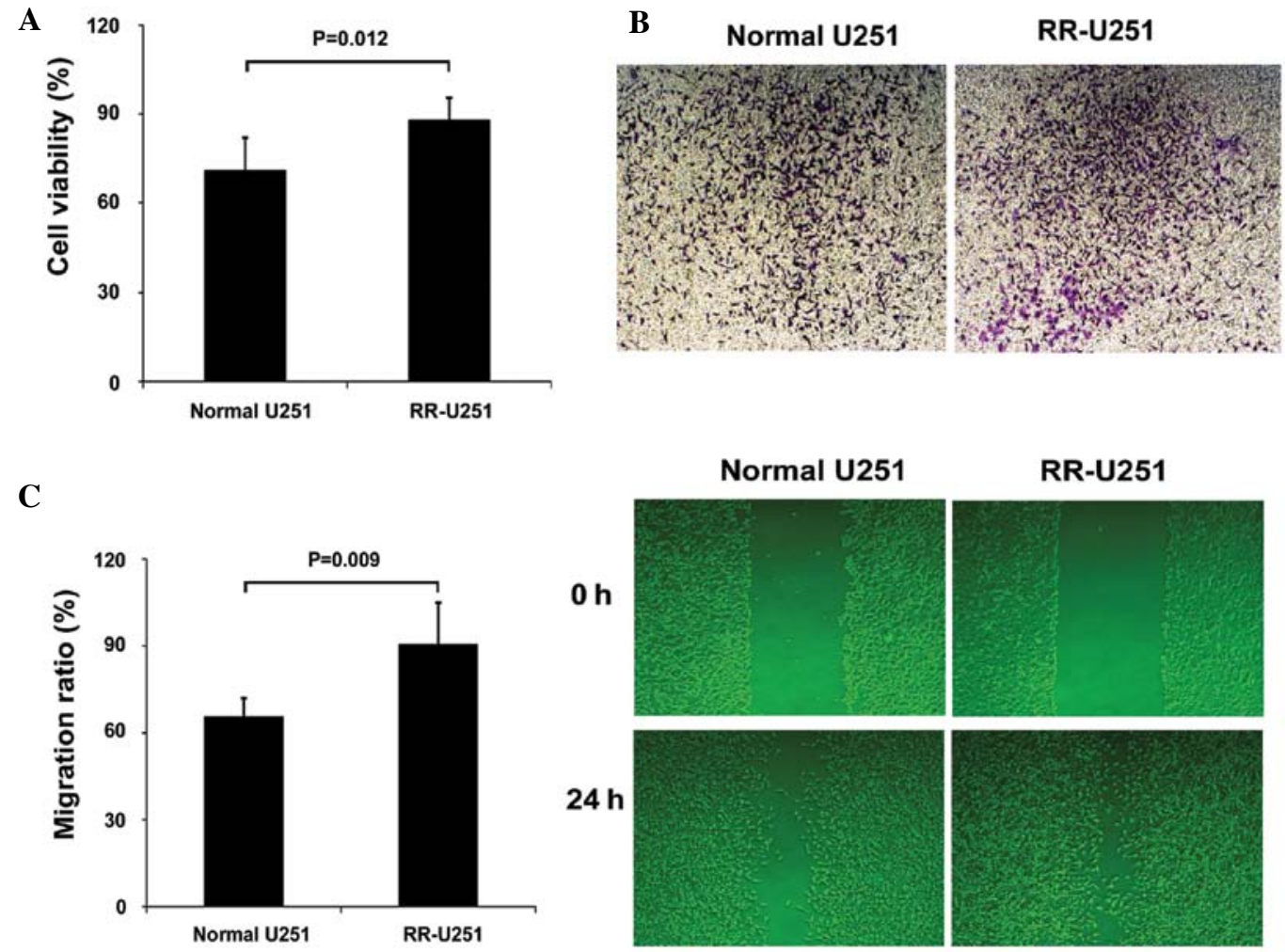

Figure 1. The radiosensitivity of normal U251 and RR-U251 cells. Following radiation treatment, the cell viability (A), invasion (B) and migration (C) abilities were measured in normal U251 and RR-U251 cells. Data are representative of three independent experiments.

at a density of $1 \times 10^{5} / \mathrm{ml}$. The $100 \mu 1$ suspension was seeded into the upper chambers. DMEM supplemented with $15 \%$ FBS was added to the lower chamber. Following incubation for $24 \mathrm{~h}$, the upper chamber was taken out and the cells remaining on the upper surface of the membrane were removed very lightly using a medical cotton bud. The invaded cells on the other side were fixed with dehydrated alcohol and stained with $0.1 \%$ crystal violet for several minutes. The chamber was viewed under a light microscope and images were captured in 5 randomly selected $\times 200$ fields for each well. Three separate experiments were performed.

Statistical analysis. The analysis was performed using SPSS 13.0. Statistical significance was analyzed using the Student's t-test and the Mann-Whitney U test. Data are expressed as the means \pm standard deviation (SD). $\mathrm{P}<0.05$ was considered to indicate a statistically significant difference.

\section{Results}

$R R-U 251$ cells and measurement of the radiosensitivity. RR-U251 cells were established from normal U251 cells, irradiated by a ${ }^{60} \mathrm{Co}$ source at $0.5 \mathrm{~Gy} / \mathrm{min}$ for $10 \mathrm{~min}$ per time until the accumulation was ${ }^{60} \mathrm{~Gy}$, and the radiosensitivity was characterized by measuring the cell viability, migration and invasion abilities after radiation treatment. The results showed that the cell viability was significantly increased in the radiation-treated RR-U251 cells, compared with radiation-treated normal U251 cells. Similar results were also observed in cell migration and invasion assays. All results suggested that the radiosensitivity was decreased in RR-U251 cells (Fig. 1).
Elevated expression of PGK1 in RR-U251 cells. The PGK1 expression was determined by real-time PCR and western blot analysis. Compared with normal U251 cells, elevated expression of both mRNA and protein levels of PGK1 was observed in RR-U251 cells (Fig. 2).

Confirmation of the transfection efficiency. Normal U251 and RR-U251 cells were transfected with shRNA-PGK1 to decrease the expression of PGK1. As shown in Fig. 3A, transfection of shRNA-PGK1 led to a stable exogenous gene expression with $\sim 50-60 \%$ efficiency in U251 cells as indicated by the GFP-labeled reporter fluorescence. We further confirmed that both normal U251 and RR-U251 cells, which were transfected with shRNA-PGK1, showed a significantly lower expression of PGK1 as compared with those transfected with an irrelevant sequence control. The cells, transfected with high expression plasmid pcDNA3.1-PGK1, also showed higher PGK1 expression as compared with controls confirmed by western blot analysis (Fig. 3B and C). We also tested the PGK1 expression 24 and $48 \mathrm{~h}$ after transfection, and found that after $24 \mathrm{~h}$ the role was more efficient (data not shown). The time-point of $24 \mathrm{~h}$ after transfection was, therefore, used in the following experiments with radiation treatment.

PGK1 modulates U251 cell proliferation. The proliferation rates of U251 cells with enhanced or decreased expression of PGK1 were determined by the MTT assay. Compared to control cells, cells transfected with shRNA-PGK1, both normal U251 and RR-U251 cells, proliferated at a significantly lower rate. In contrast, overexpression of PGK1 by infection with high expression plasmid pcDNA3.1-PGK1 resulted in significantly 
A

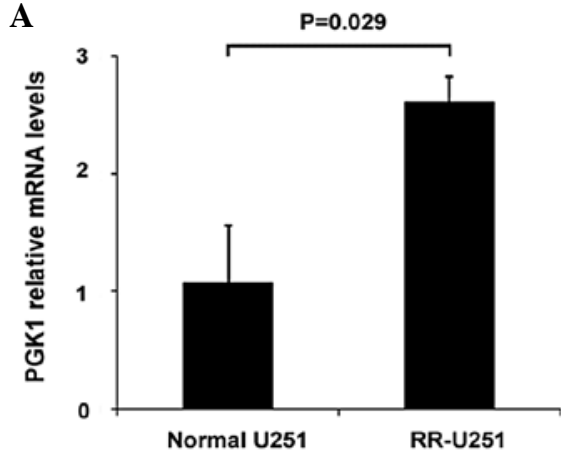

B

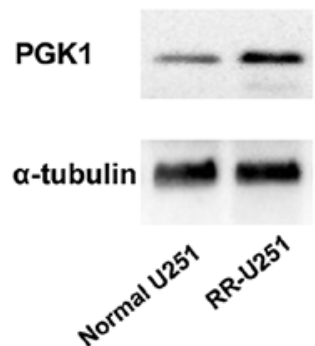

C

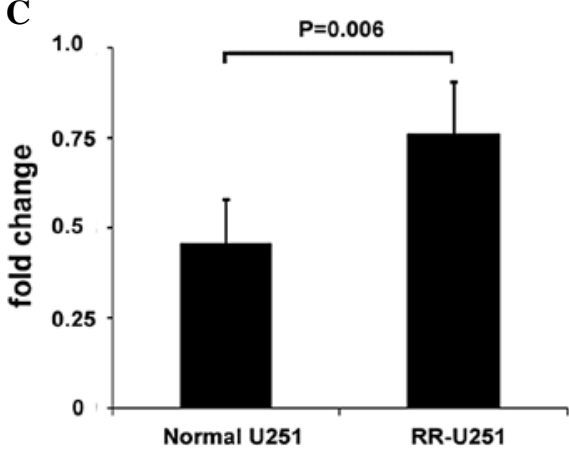

Figure 2. PGK1 expression in normal U251 and RR-U251 cells. Real-time PCR was performed to evaluate the mRNA expression of PGK1. (A) Results were presented as fold increases relative to the expression of human GAPDH. (B) Western blot analysis was used to detect the protein expression of PGK1. $\alpha$-tubulin served as the loading control. (C) Relative expression of PGK1 was determined by densitometry. Data are representative of three independent experiments.

A
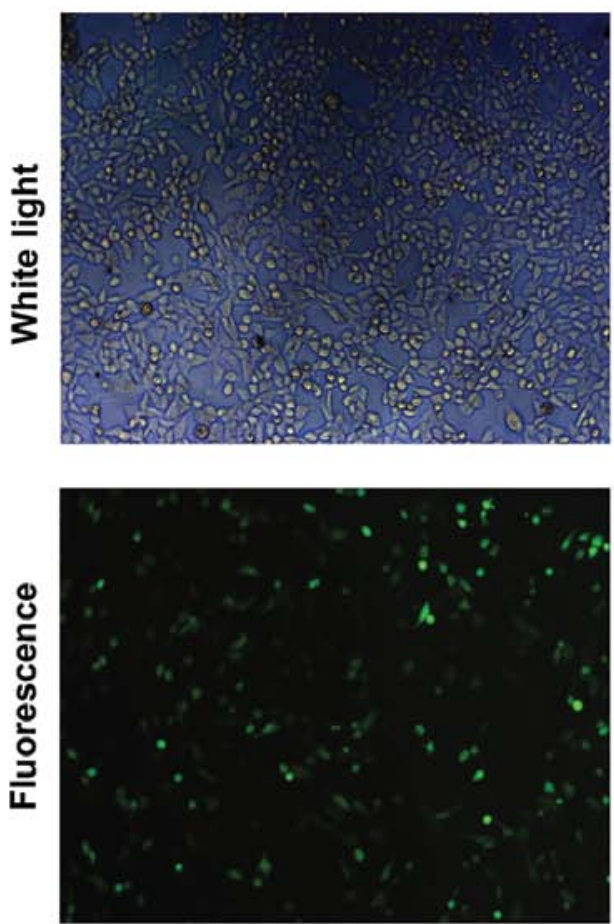

B

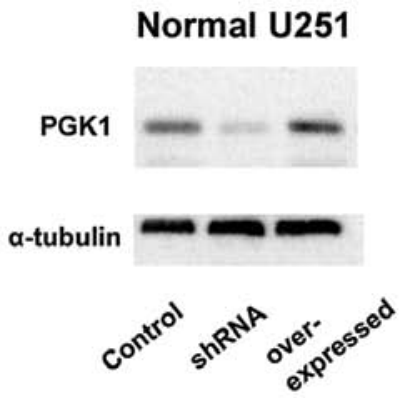

C

RR-U251

PGK1

a-tubulin
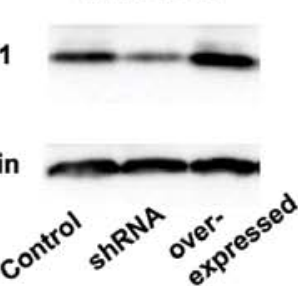

D

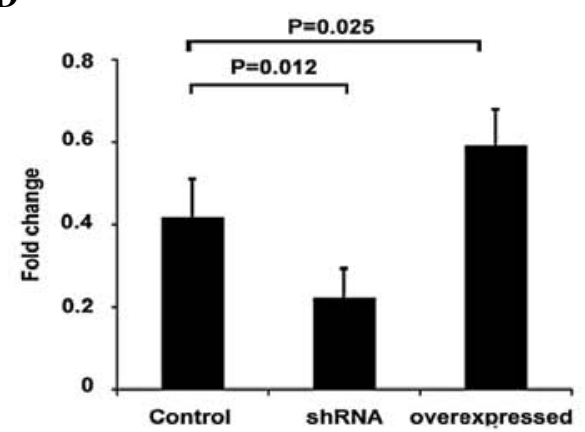

E

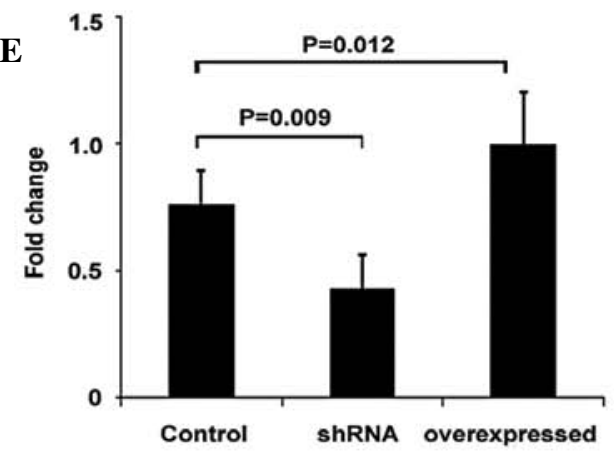

Figure 3. Modulating PGK1 expression by transfection. (A) U251 cells transfected with GFP-labeled shRNA-PGK1 were observed by inverted fluorescent microscopy. PGK1 protein expression in normal U251 (B) and RR-U251 (C) cells $24 \mathrm{~h}$ after transfection with shRNA-PGK or pcDNA3.1-PGK1 was analyzed by western blot analysis. $\alpha$-tubulin served as the loading control. Relative expression of PGK1 was determined by densitometry in normal U251 (D) and RR-U251 (E) cells. Data are representative of three independent experiments.

enhanced proliferation in both normal U251 and RR-U251 cells. We further downregulated PGK1 expression in PGK1 overexpressed U251 cells, using shRNA-PGK1 transfection. The shRNA-PGK1-transfected cells showed significantly lower proliferation rate compared with the control group, both in normal U251 and RR-U251 cells (Fig. 4).

PGK1 modulates U251 cell migration ability. The migration ability of U251 cells with enhanced or decreased expression of PGK1 was determined by the WHA method. Following radiation therapy, both shRNA-PGK1-transfected normal U251 and RR-U251 cells showed significantly decreased migration abilities, compared with control cells. In contrast,
PGK1-overexpressed cells showed significantly enhanced migration abilities in both normal U251 and RR-U251 cells. We also used shRNA-PGK1 transfection to further downregulate PGK1 expression in PGK1-overexpressed U251 cells. The migration ability of PGK1-overexpressed cells was significantly decreased after transfection with shRNA-PGK1, in both normal U251 and RR-U251 cells (Fig. 5).

PGK1 modulates U251 cell invasion ability. We also assessed the role of PGK1 in cell invasion by Transwell assay. As shown in Fig. 6, compared with control cells, the invasion potential of U251 cells transfected with shRNA-PGK1 was significantly decreased both in normal U251 and RR-U251 cells, while 

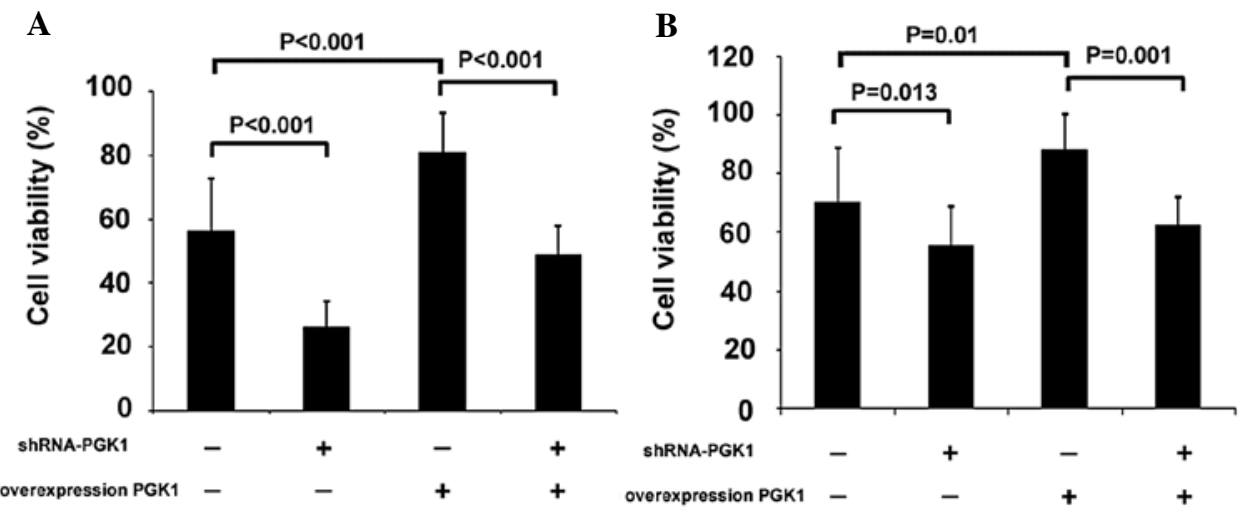

Figure 4. Effect of PGK1 on U251 cell proliferation. (A) Normal U251 and (B) RR-U251 cells, which were treated differently as indicated (control group, or shRNA-PGK1 group, or pcDNA3.1-PGK1 group, or the combined pcDNA3.1-PGK1 with shRNA-PGK1), were radiated by ${ }^{60} \mathrm{Co}$ once. After $48 \mathrm{~h}$, the proliferation ratio was measured by MTT. Data are representative of three independent experiments.

A

Control

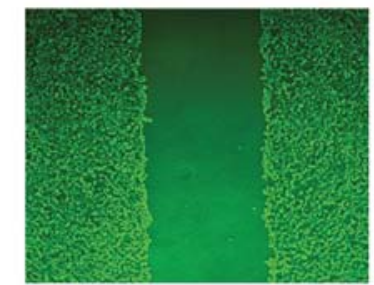

$24 \mathrm{~h}$

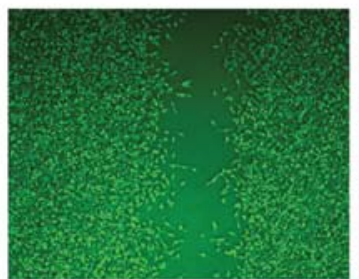

Control

B
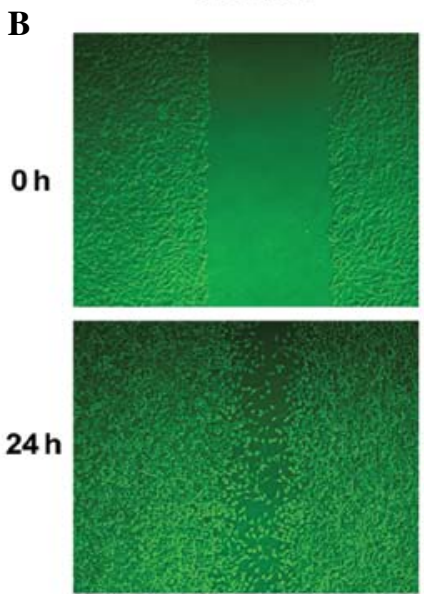

shRNA PGK1
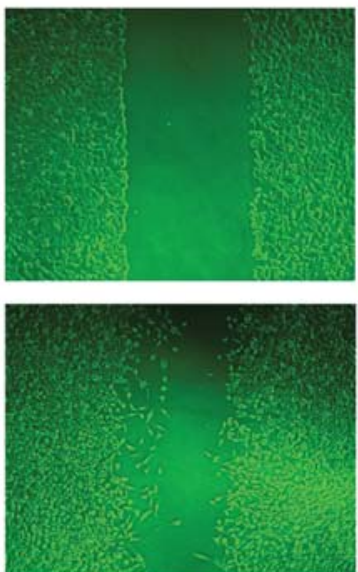

ShRNA PGK1
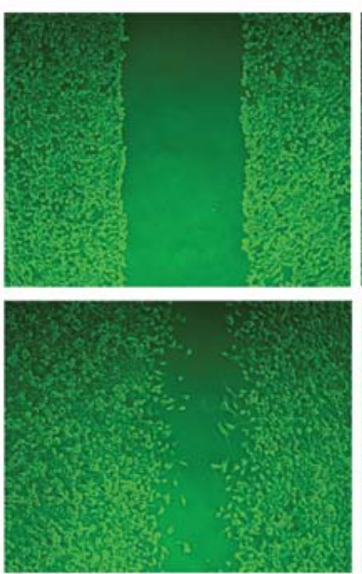

\section{Overexpressed PGK1}
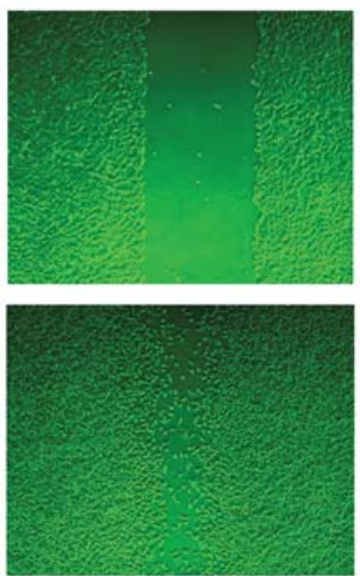

Overexpressed PGK1
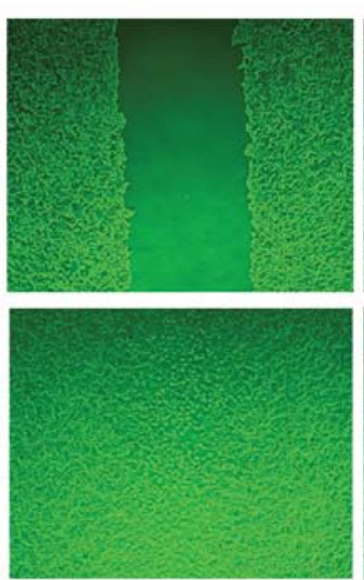

Overexpressed +shRNA PGK1
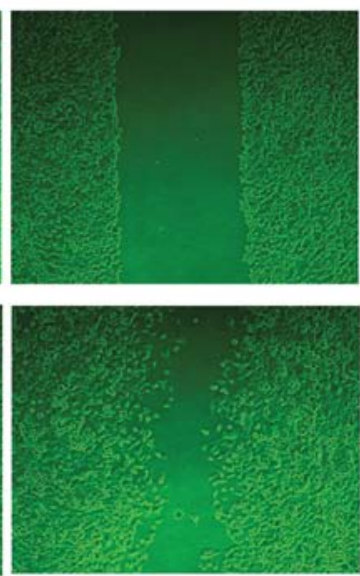

Overexpressed +shRNA PGK1
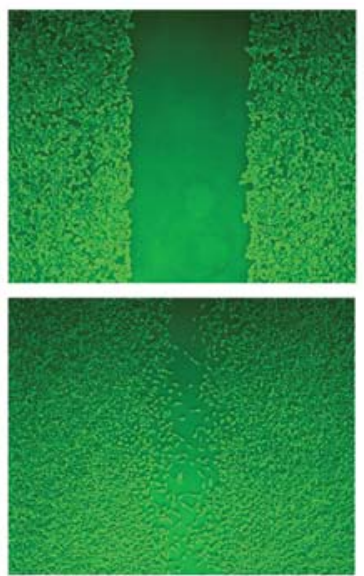

Figure 5. Effect of PGK1 on U251 cell migration ability. (A) Normal U251 and (B) RR-U251 cells, which were treated differently as indicated (control group, or shRNA-PGK1 group, or pcDNA3.1-PGK1 group, or the combined pcDNA3.1-PGK1 with shRNA-PGK1), were radiated by ${ }^{60} \mathrm{Co}$ once. The monolayer cells were scratched out using the tip of a $10 \mu \mathrm{l}$ pipette to create the wound line. Images were captured with a microscope at 0 and $24 \mathrm{~h}$ at the same site.

cells transfected with pcDNA3.1-PGK1 displayed markedly increased invasive ability. Following transfection with shRNA-PGK1, the invasion ability of PGK1-overexpressed
U251 cells also decreased compared with the control group. These results suggest that PGK1 modulates U251 cell invasion (Fig. 6). 


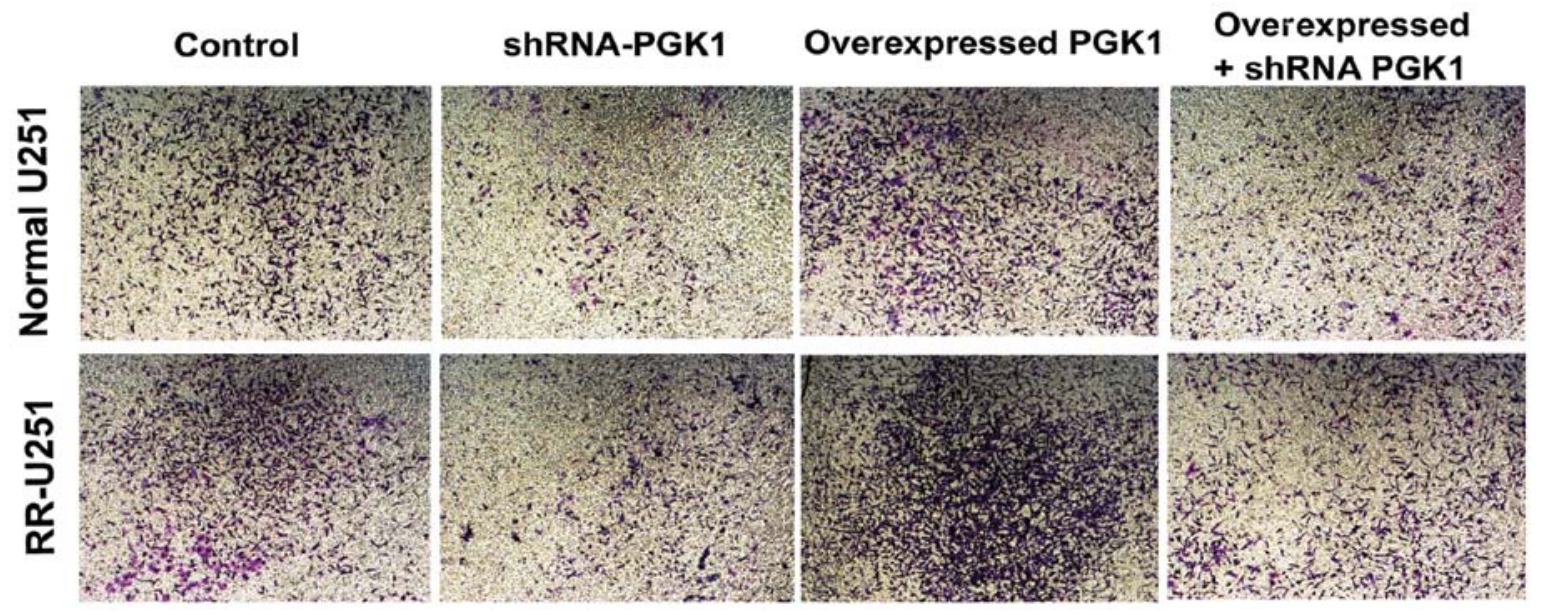

Figure 6. Effect of PGK1 on U251 cell invasion ability. Normal U251 and RR-U251 cells, which were treated differently as indicated (control group, or shRNA-PGK1 group, or pcDNA3.1-PGK1 group, or the combined pcDNA3.1-PGK1 with shRNA-PGK1), were radiated by ${ }^{60} \mathrm{Co}$ once. The invasion ability was evaluated by a Transwell culture chamber system.

\section{Discussion}

In the present study, we first observed that the expression of PGK1 was upregulated in RR-U251 cells, which are resistant to radiation. Further study confirmed that cell viability and cell migration were much higher, and cells invaded more when PGK1 was overexpressed, while blocking PGK1 in the cell viability, cell migration and invasion assay revealed significant inhibition of these parameters, suggesting that PGK1 was involved in the radioresistance in human glioma by promoting the tumorigenic properties, including cell viability, cell migration and invasion ability.

Our previous study showed that patients with radioresistant astrocytomas, compared to patients with radiosensitive astrocytomas, displayed much higher protein level of the glycolytic enzyme PGK1 (11). We proposed that PGK1 was involved in the radioresistance in glioma. U251 cells are glioma cancer cells that can induce highly aggressive tumors of the central nervous system (22) and they are an accepted model to study the biology of gliomas (23). PGK1 is a metabolismrelated ATP-generating glycolytic enzyme overexpressed in a number of malignancies and generally correlates with poor survival $(14,24,25)$. It catalyzes the reversible conversion of 1,3-diphosphoglycerate to 3-phosphoglycerate and further affects DNA replication and repair (12). Increasing glycolysis in radioresistant astrocytomas suggests a status of hypoxia. In a range of human tumor sites, the presence of hypoxia is a negative prognostic indicator for outcome following radiotherapy (26-28). As is well known, hypoxia plays a central role in the pathobiology of glioma (9). The key mediator, the transcription factor hypoxia inducible factor 1 (HIF-1), is stabilized under hypoxic conditions and promotes the production of vascular endothelial growth factor (VEGF), which is critical for neovascularization. Hypoxia-driven physiologic changes also include the glycolytic pathway regulation and bloodvessel formation (29) and also promote glioma cell migration and invasion (30). PGK1, a key enzyme of anaerobic glycolytic metabolism, is also one of the target genes of HIF-1 (31), and we found that PGK1 was upregulated in both radioresistant astrocytomas and RR-U251 cells. Therefore, we speculate that in glioma conditions, hypoxia augments HIF-1 secretion and consequently affects PGK1 expression, resulting in resistance of glioma radiotherapy. This hypothesis is in general agreement with that reported by Lam et al (32), who found that the induction of HIF-1 in hypoxic conditions can induce the expression of PGK in non-small cell lung carcinoma cells. We hypothesized that a vicious circle may arise between increased PGK1 and radioresistance in glioma.

On the other hand, in the present study, our new data concerning PGK1 provided comprehensive evidence of the Warburg effect, a phenomenon first described more than 80 years ago that cancer cells maintain a high glycolytic rate even in the presence of oxygen (33). To explain the conversion of glucose to lactate by cancer cells in the presence of oxygen, Warburg speculated that tumor mitochondria are decreased or functionally impaired. Previous studies renewed the Warburg effect and clarified its exact role as cause, correlate, or facilitator of cancer $(34,35)$. Meanwhile it is largely accepted and shown that solid tumor cells employ glycolytic enzymes, such as PGK1, to produce ATP when their supply of oxygen is limited (36). Furthermore, PGK1 has been shown to be overexpressed in several malignancies, such as metastatic colon cancer (13), lung adenocarcinoma (14), gastric (15), prostate (16), pancreatic (17) and ovarian cancer (18-19). Meanwhile, our results were generally consistent with a recent study performed on ovarian cancer, in which siRNA-PGK1 sensitized chemoresistant human ovarian cancer cell lines to cisplatin (37). These findings support the comprehension between glucose metabolism and cancer generation and may serve as a potential therapeutic target to prevent metastasis of cancer cells. Our results presented here suggest that PGK1 could promote radioresistance in human U251 cells, and manipulation of PGK1 may offer a novel therapeutic approach for the treatment of glioma.

Interest in cancer stem cells is rapidly increasing. In glioma, studies have shown that stem cells could promote radioresistance by preferential activation of the DNA damage response (38). Whether PGK1 plays a potential role in regulating radioresistance of glioma stem cells remains unclear and further studies are required to clarify this issue. 
Our previous study showed that patients with radioresistant astrocytomas, compared to patients with radiosensitive astrocytomas, displayed much higher protein level of PGK1 (11). However, the results are a long way from using the levels of PGK1 in human gliomas as a potential marker for predicting the radioresistance in clinical application. More glioma cell lines should be included to confirm the role of PGK1 in the glioma. Further studies using PGK1 neutralization or PGK1 knockout mice are needed to examine the exact role of PGK1 in the radioresistance of glioma animal models. Also, the relationship between PGK1 levels and the survival rate after radiotherapy in glioma patients (radioresistant or nonradioresistant) should also be investigated prospectively. This knowledge is necessary before a role of PGK1 in the treatment of glioma can be envisaged.

\section{Acknowledgements}

The present study was supported by the grant NSFC 81172390 from the National Science Foundation of China and the grant ZKX10021 from the Health Bureau of Nanjing.

\section{References}

1. Cancer incidence in five continents. Volume VIII. IARC Sci Publ pp1-781, 2002.

2. Schwartzbaum JA, Fisher JL, Aldape KD and Wrensch M: Epidemiology and molecular pathology of glioma. Nat Clin Pract Neurol 2: 494-503, 2006.

3. Maher EA, Furnari FB, Bachoo RM, et al: Malignant glioma: genetics and biology of a grave matter. Genes Dev 15: 1311-1333, 2001.

4. Stupp R, Mason WP, van den Bent MJ, et al: Radiotherapy plus concomitant and adjuvant temozolomide for glioblastoma. N Engl J Med 352: 987-996, 2005.

5. Krakstad C and Chekenya M: Survival signalling and apoptosis resistance in glioblastomas: opportunities for targeted therapeutics. Mol Cancer 9: 135, 2010.

6. Poonnoose SI and Daniel RT: Radiological evidence of glioma invasion of the central nervous system along tracts. Surg Neurol 54: 194-196, 2000.

7. Tate MC and Aghi MK: Biology of angiogenesis and invasion in glioma. Neurotherapeutics 6: 447-457, 2009.

8. Grossman SA and Batara JF: Current management of glioblastoma multiforme. Semin Oncol 31: 635-644, 2004

9. Preusser M, de Ribaupierre S, Wohrer A, et al: Current concepts and management of glioblastoma. Ann Neurol 70: 9-21, 2011.

10. Zinn PO, Colen RR, Kasper EM and Burkhardt JK: Extent of resection and radiotherapy in GBM: A 1973 to 2007 surveillance, epidemiology and end results analysis of 21,783 patients. Int J Oncol 42: 929-934, 2013.

11. Yan H, Yang K, Xiao H, Zou YJ, Zhang WB and Liu HY: Over-expression of cofilin-1 and phosphoglycerate kinase 1 in astrocytomas involved in pathogenesis of radioresistance. CNS Neurosci Ther 18: 729-736, 2012.

12. VandeBerg JL: The phosphoglycerate kinase isozyme system in mammals: biochemical, genetic, developmental, and evolutionary aspects. Isozymes Curr Top Biol Med Res 12: 133-187, 1985.

13. Ahmad SS, Glatzle J, Bajaeifer K, et al: Phosphoglycerate kinase 1 as a promoter of metastasis in colon cancer. Int $\mathbf{J}$ Oncol 43: 586-590, 2013

14. Chen G, Gharib TG, Wang H, et al: Protein profiles associated with survival in lung adenocarcinoma. Proc Natl Acad Sci USA 100: 13537-13542, 2003.

15. Zieker D, Konigsrainer I, Tritschler I, et al: Phosphoglycerate kinase 1 a promoting enzyme for peritoneal dissemination in gastric cancer. Int J Cancer 126: 1513-1520, 2010.
16. Wang J, Ying G, Jung Y, et al: Characterization of phosphoglycerate kinase-1 expression of stromal cells derived from tumor microenvironment in prostate cancer progression. Cancer Res 70: 471-480, 2010.

17. Cecconi D, Palmieri M and Donadelli M: Proteomics in pancreatic cancer research. Proteomics 11: 816-828, 2011.

18. Duan Z, Lamendola DE, Yusuf RZ, Penson RT, Preffer FI and Seiden MV: Overexpression of human phosphoglycerate kinase 1 (PGK1) induces a multidrug resistance phenotype. Anticancer Res 22: 1933-1941, 2002.

19. Lincet H, Guevel B, Pineau C, et al: Comparative 2D-DIGE proteomic analysis of ovarian carcinoma cells: toward a reorientation of biosynthesis pathways associated with acquired platinum resistance. J Proteomics 75: 1157-1169, 2012.

20. Lin TY, Chang JT, Wang HM, et al: Proteomics of the radioresistant phenotype in head-and-neck cancer: Gp96 as a novel prediction marker and sensitizing target for radiotherapy. Int J Radiat Oncol Biol Phys 78: 246-256, 2010.

21. Livak KJ and Schmittgen TD: Analysis of relative gene expression data using real-time quantitative PCR and the 2(-Delta Delta C(T)) method. Methods 25: 402-408, 2001.

22. Rich JN and Bigner DD: Development of novel targeted therapies in the treatment of malignant glioma. Nat Rev Drug Discov 3: 430-446, 2004

23. Nakamizo A, Marini F, Amano T, et al: Human bone marrowderived mesenchymal stem cells in the treatment of gliomas. Cancer Res 65: 3307-3318, 2005.

24. Hwang TL, Liang Y, Chien KY and Yu JS: Overexpression and elevated serum levels of phosphoglycerate kinase 1 in pancreatic ductal adenocarcinoma. Proteomics 6: 2259-2272, 2006.

25. Fantin VR, St-Pierre J and Leder P: Attenuation of LDH-A expression uncovers a link between glycolysis, mitochondrial physiology, and tumor maintenance. Cancer Cell 9: 425-434, 2006.

26. Brizel DM, Dodge RK, Clough RW and Dewhirst MW: Oxygenation of head and neck cancer: changes during radiotherapy and impact on treatment outcome. Radiother Oncol 53: 113-117, 1999.

27. Fyles AW, Milosevic M, Wong R, et al: Oxygenation predicts radiation response and survival in patients with cervix cancer. Radiother Oncol 48: 149-156, 1998.

28. Knocke TH, Weitmann HD, Feldmann HJ, Selzer E and Potter R: Intratumoral $\mathrm{pO}_{2}$-measurements as predictive assay in the treatment of carcinoma of the uterine cervix. Radiother Oncol 53: 99-104, 1999

29. Semenza GL: Defining the role of hypoxia-inducible factor 1 in cancer biology and therapeutics. Oncogene 29: 625-634, 2010.

30. Fujiwara S, Nakagawa K, Harada $\mathrm{H}$, et al: Silencing hypoxiainducible factor- $1 \alpha$ inhibits cell migration and invasion under hypoxic environment in malignant gliomas. Int J Oncol 30: 793-802, 2007.

31. Semenza GL, Roth PH, Fang HM and Wang GL: Transcriptional regulation of genes encoding glycolytic enzymes by hypoxiainducible factor 1. J Biol Chem 269: 23757-23763, 1994.

32. Lam W, Bussom S and Cheng YC: Effect of hypoxia on the expression of phosphoglycerate kinase and antitumor activity of troxacitabine and gemcitabine in non-small cell lung carcinoma. Mol Cancer Ther 8: 415-423, 2009.

33. Warburg O: On the origin of cancer cells. Science 123: 309-314, 1956.

34. Kim JW and Dang CV: Cancer's molecular sweet tooth and the Warburg effect. Cancer Res 66: 8927-8930, 2006.

35. Vander Heiden MG, Cantley LC and Thompson CB: Understanding the Warburg effect: the metabolic requirements of cell proliferation. Science 324: 1029-1033, 2009.

36. Daly EB, Wind T, Jiang XM, Sun L and Hogg PJ: Secretion of phosphoglycerate kinase from tumour cells is controlled by oxygen-sensing hydroxylases. Biochim Biophys Acta 1691: 17-22, 2004.

37. Lepleux C, Abeilard-Lemoisson E, Duval M, Icard P and Lincet H: siPGK1 sensitizes chemoresistant human ovarian cancer cell lines to cisplatin. Anticancer Res 32: 4277-4286, 2012.

38. Bao S, Wu Q, McLendon RE, et al: Glioma stem cells promote radioresistance by preferential activation of the DNA damage response. Nature 444: 756-760, 2006. 